\title{
Digitizing Interaction: The Application of Parameter-Oriented Design Methodology to the Teaching/ Learning of Interaction Design
}

\author{
Shu-Wen Tzeng \\ Department of Industrial and Graphic Design, Auburn University, \\ Auburn, Alabama 36849, USA \\ szt0004@auburn. edu
}

\begin{abstract}
The development of digital technology has changed the way users interact with products and forced industrial design educators to rethink the role of design education with respect to both the integrity and suitability of current design curriculum. This study is sought to figure out a better way for teaching/ learning Interaction Design in the discipline of Industrial Design with considerations to the nature of interaction design and students' learning mode. A newly created interaction design methodology will be introduced in this paper, and the case study on the application of this approach to a graduate school level interaction design course should explain how this methodology can be manipulated in the development of an interaction design, making teaching/ learning Interaction Design more effective and enjoyable.
\end{abstract}

Keywords: Interaction Design, Design Methodology, Design Education.

\section{Introduction}

In his article "Tomorrow's Design Landscape: Anywhere Interactions", Bill Mak, an innovation interaction-design entrepreneur and the chair of IDSA's Interaction Design Professional Interest Section, stated that “ Three possibilities for innovation- mobile high-speed Internet, contextual awareness and anywhere physical interactions- will change the world and professional design practice." He also foretold, " The third possibility suggests that digital convergence will not just take interaction design to a dramatically broader industrial ecosystem of electronics, machines, objects and devices but also promote the digital evolution of many traditionally non-digital product areas." [1]. To date, the development of digital technology has opened many opportunities to integrate diverse technologies and new interaction paradigms into many categories of products and may promote innovations in many non-digital products. Hence, more and more industrial designers of digital technology products no longer regard their jobs as designing a physical object but as designing the interactions between the user and the product. In other words, the boundary between hardware and software has become blurred and the demand for a good user- product interaction has never been so acute. 
In school, the changes in technology are also forcing design educators to rethink the role of design education with respect to both the integrity and suitability of current design curriculum. It is apparent that we are now at the leading edge of a wave of technological change that will affect all aspects of everyday life in a profound way. The next generation of designers will need new skills and knowledge to negotiate this new terrain. As the focus of product design has shifted from the physical functionality and aesthetics to user interfaces, and finally to the ubiquitous interactive paradigms, industrial design students must have a solid appreciation of Interaction Design topics to succeed. Understanding Interaction Design will allow designers to create products that are usable by everyone, extending the impact of user-product interaction to a diverse set of users within many domains. Yet industrial design educators are now struggling with teaching Interaction Design due to the lack of methods, which adapt to students' learning mode (in this case, the Net Generation's learning mode) and are easy-to learn and quick to examine. Because the design of interaction interface often depends on both the author's thinking and the presumed audience's experience, educators must endow their students with abilities to solve problems by using procedures and analytic methods. The solution to this problem relies on educators' extensive study on Interaction Design and continuous attempts at creating effective teaching strategies. Therefore, this study is sought to figure out a better way for teaching/ learning Interaction Design in the discipline of Industrial Design. The goal is to equip the next generation of industrial designers with the abilities to tackle human's substantial needs of interaction.

\section{Interaction Design in the Field of Industrial Design}

\subsection{The Nature of Interaction Design}

According to Bill Moggridge, who coined the term "interaction design" together with Bill Verplank in the late 1980s, Interaction Design is the study of devices with which a user can interact [2]. In other words, Interaction Design defines the behavior (the "interaction") of an artifact or system in response to its users. Because the behavior of an artifact is often embodied in both the abstract content and the concrete form of an interface design, it makes sense to assume that any interaction design is composed of user, content and form of design, as shown in Figure 1.

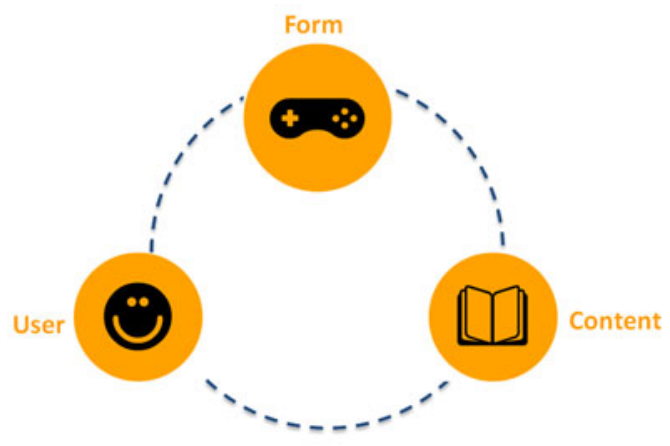

Fig. 1. The three main components in an interaction design 
These three components- the user, the content, and the form of design- are both independent from and correlated with each other- the content and the form of design can influence the users' experience and productivity while have no power to change the users' profiles or intentions. The user, with no doubt, is the center of an interaction design since the ultimate goal of Interaction Design is to create a great user experience. Note that the user consideration here is more focused on users' intentions rather than users' needs -- the way a user chooses to interact with an interface is often determined by his/ her purpose or intentions in accessing the content. The second consideration, the content, can be referred to the services provided by an artifact. The content is collected and delivered through different ways based on the pre-determined functions. Because Interaction Design is often looking at the user's productivity rather than that of the product, it is more interested in the delivery strategy of the content instead of the content itself. In their design research, Blair-Early and Zender find that the type of delivery of content is one of the most important factors contributing to the quality of an interaction design and has more power to influence end-user experience comparing to the content itself [3]. The form of design, which is often referred to an interface, is the means by which users interact with content for a purpose. The form can be presented through different media- from a physical object to a gesture-oriented interaction, and exists in various kinds of products in our everyday life. As the number of interfaces and the diversity of users glow, the need for effective interaction design increases. Therefore, the form of design has gradually become the main focus in an interaction design. As interaction design becomes a new terrain for industrial designers, the educators are under obligation to teach interaction design to their students in an effective way.

\subsection{Teaching Interaction Design to Industrial Design Students}

Similar to other interaction design related disciplines, the interaction design curriculum in industrial design begins with a design research, which investigates user, process, cultural context, form, and content, and follows with an iterative design process including idea exploration, design execution and design validation. Technically, this is a process from collecting abstract information (often involving the understanding of user behaviors and goals), to defining content functions (within a conceptual framework and with an emphasis on the values of the content), and finally to constructing the concrete structure of a user interface design. This process can be presented in the structure as shown in Figure 2.

Different from other disciplines, industrial design has more focus on the communications between the user and the artifact through the form of design- often in the fashion of visual display of quantitative information and visual explanations. It has been believed that the "design principles" can help designers achieve an effective interface [3], [4], [5], [6], [7], [8]. Hence, tons of interaction design professionals have attempted to improve interface design primarily by exploring and analyzing existing form (patterns) of interface design, or by providing design principles to guide the design of interaction. Two of the most famous examples are the 1992 Version of "Macintosh Human Interface Guidelines" published by Apple Computer, which suggests thirty-eight index entries for icons in an interface design [4], and the Nielsen 


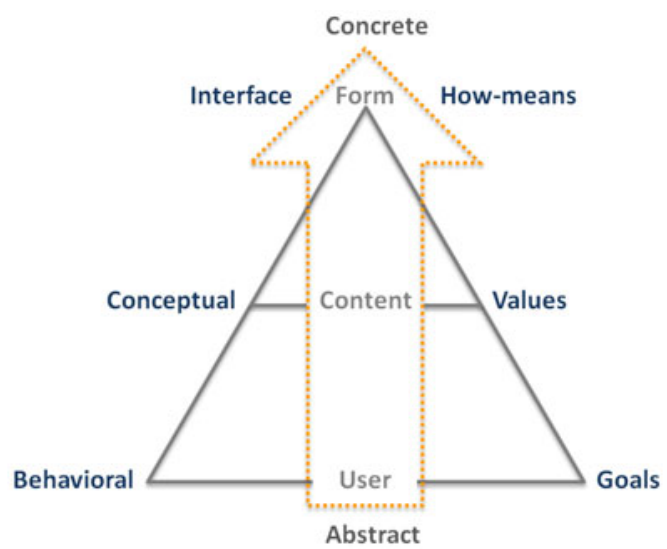

Fig. 2. The general process and factors in an interaction design

Norman Group's 106-page report, "Site Map Usability”, which delivers twenty-eight guidelines " to improve an interaction design" [9].

These design principles have two problems: they are too vague to be usefulespecially for design students or junior designers; and, as final experiences, they provide no indication of how they may be achieved. Therefore, there is a need for the design educators to figure out a better way to teach interaction design, especially on how design principles can be applied to an interaction design. What follows is a description of the proposed design methodology that has been used for teaching/ learning interaction design since 2008. This design method is created with the considerations of students' learning mode and ease of examination for future design validation, making learning more efficient and effective.

\section{The Idea of Parameter-Oriented Design Methodology}

The Parameter-Oriented Interaction Design Methodology is partly inspired by the parameter concept proposed by Blair-Early and Zender [3], and partly inspired by the digital technology which is extensively utilized in the everyday life of current college students.

Many current university students belong to the 'Net Generation'- a label used to describe today's young adults. This group of individuals, born between 1980 and 1994 [10], has been characterized by their familiarity with and reliance on digital technologies. A number of social psychologists have argued that the digital culture in which the Net Generation have grown up has influenced their preferences and skills in a number of key areas related to education. For example, the Net Generation are said to prefer receiving information quickly; expect immediate answers; prefer multi-tasking and non-linear access to information; rely heavily on communications technologies to access information and to carry out social and professional interactions [11], [12]. Compare to analog signal, digital signal is easier to recognize, define, and manipulate, meeting the needs of Net Generation in terms of simplicity and speed [13]. The digital signal takes the form of parameter, which is a computation from recorded data, to 
present its relative value in a system. Therefore, the Parameter-Oriented Design Methodology should be an ideal way for teaching/ learning interaction design.

In their research on identifying user interface design principles for interaction design, Blair-Early and Zender propose a set of "parameters" for achieving an effective interface. They believe that principles in isolation do not provide sufficient guidance to inform design decision, only when parameters and principles working together can drive innovation and empower designers. Based on the three components model for an interaction design, Blair-Early and Zender define the "parameters" that govern an effective interface as:

User Intention: Hunter-Browser.

Content Delivery: Reference- Educational- Inspiration- Entertainment. Interface Type: Linear-Hierarchy-Matrix-Web.

Note that the Interface Type here can only be referred to the structure of an interface design rather than the form of an interaction design; hence, only the parameters defined for user and content are considered in this design methodology. The descriptions of these parameters are shown as below:

User Intention. The Hunter: the hunter is focused, precise and often destinationdriven. The hunter values the speed and efficiency of an interface, and rarely deviates from its initial content direction to discover a new path. A hunter may scan large quantities of information quickly in order to find a predetermined target information or content. The Browser: the browser is intent on the journey and, in many cases, may not have a final content destination in mind. The browser is less focused and driven in the search for content, and more likely to be open to new experiences.

Content Delivery Strategy. Reference: a content delivery strategy designed to serve discrete bits of information to users. The reference source is driven to provide as much information as possible in as few steps as possible. Educational: a content strategy designed to instruct, often in a step-by-step fashion. Educational content is driven to educate its audience. Inspiration: a content strategy designed to motivate or inspire. Often, the inspirational source has a more personal connection to the audience through calls to action and directives. The inspirational source derives its trust through emotional response and personal connection rather than through factual data. Entertainment: an entertainment delivery strategy is designed to amuse and geared to draw a browser audience. It establishes a more direct connection with the audience and requires direct participation from the user. Entertainment sources are the most open to interpretation, and may even require audience participation in establishing the content.

It is important for designers to define these parameters before working on design exploration. Because these parameters are derived from research results, identifying design parameters can help designers clarify some essential questions. Once these parameters are identified, designers can define the level of interaction based on their knowledge of user intentions and content delivery strategies by applying appropriate design principles to their design, making interaction design more effective and relevant. Note that the level of interaction/ the desired experience is the key in this design methodology; it should be considered within each single design principle. Therefore, the structure of the Parameter-Oriented Design Methodology can be presented in the following figure (Figure 3 ): 


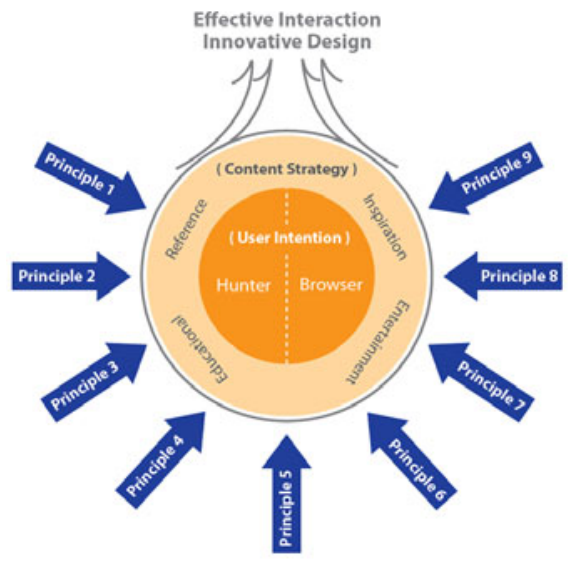

Fig. 3. The structure of the Parameter-Oriented Design Methodology

At the very center of this model is the user intention, which can be classified into two parameters- the hunter and the browser, and follows the consideration of content strategy, which is divided into four main types: the reference, the educational, the inspiration, and the entertainment. All of the design principles are used to guide design decisions by defining the appropriate level of interaction in the fashion of digital parameters. Take "the principle of feature exposure" and "the principle of user autonomy" for examples; to define the form of an interaction design for a browsing user experiencing entertainment content relies on the understanding of both user intention and content strategy. Based on the gathered information, it is obvious that intensive feature exposure is important and more authorship is required to achieve an effective interaction design within this kind of scenario. Therefore, if these design elements can be presented in a scale composed of nine numbers - from 1 to 9, the design decisions can be illustrated on the following chart:

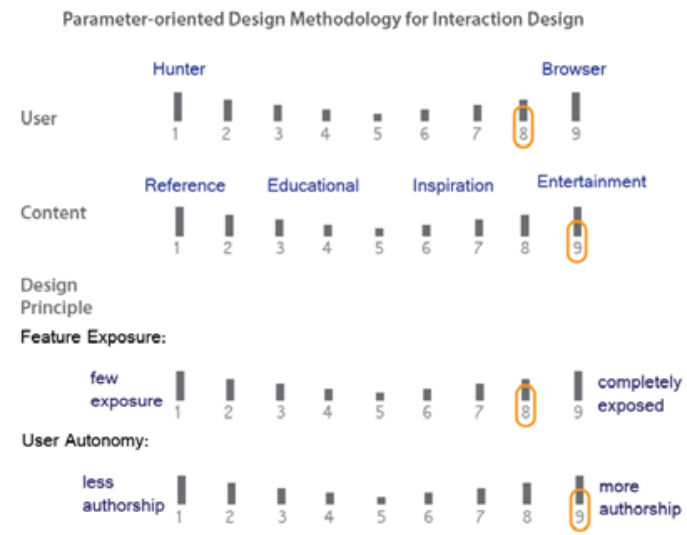

Fig. 4. The design parameters identified by interaction designers based on the considerations of user intention, content strategy, and level of interaction 
Note that the decisions related to "Feature Exposure" and "User Autonomy" align roughly with the interaction parameters above them, causing each design decision to be taken in reference to the specific considerations being addressed. This process will be followed with each design principle in an iterative fashion. In practice, consideration of just a few principles generally leads to a design theme or system that encompasses the other principles. With the great flexibility in accounting for all the relevant factors in an interaction design, the Parameter-Oriented Design Methodology is both easy to practice and inviting invention and innovations. Recipes for making an interaction design are replaced by guidance in establishing landmarks and contexts for a particular user intention and content strategy- making the teaching/ learning of interaction design more effective and enjoyable.

\section{The Application of Parameter-Oriented Design Methodology to the Teaching/ Learning of Interaction Design}

\subsection{The Execution of Parameter-Oriented Design Methodology in Design Projects}

This paragraph describes how the Parameter-Oriented Design Methodology was applied to a social networking device design conducted by two industrial design graduate students. The Friend Finder was designed for a hunter persona within a reference interface concept. Based on students' understanding of user intention and content strategy, the result of defining design parameters can be graphically illustrated below:

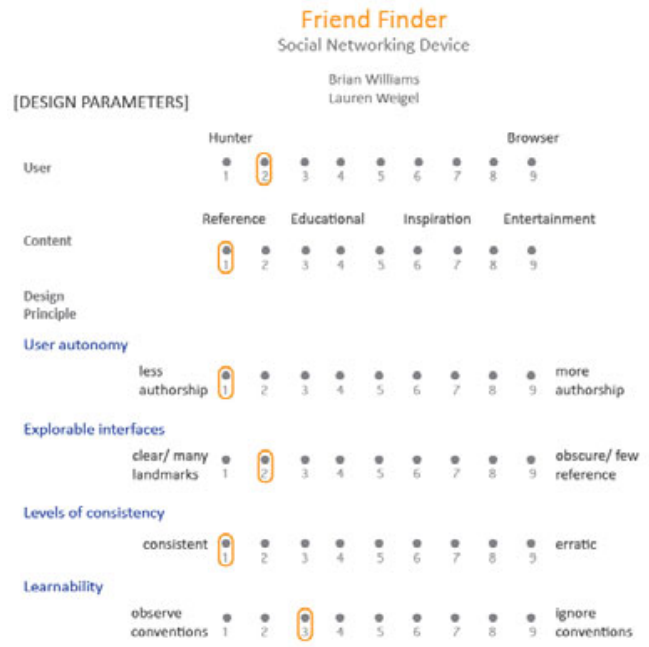

Fig. 5. Student example, Brian Williams and Lauren Weigel. Design parameters were placed within a matrix based on what user intention and content strategy are and how closely they related to the search query. 
The goal of this project was to develop features and systems within a person's mobile ecosystem that better enables them to manage a connection to information and others in order to create more meaningful destinations. Because the hunter requires immediate information with the least amount of input and the reference device is aimed to give the user a way of navigating and connecting within their social network, the design parameters consist of relatively low user autonomy, tons of clear landmarks with consistent design language, and conventional visual metaphors. Some interface designs of this project are presented in Figure 6.

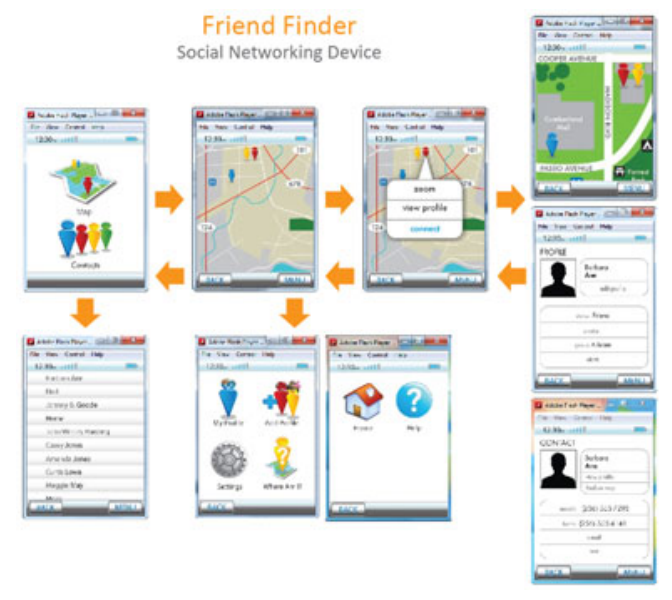

Fig. 6. Student example, Brian Williams and Lauren Weigel. Friend Finder interaction design and process of use.

\subsection{Educational Result and Student Benefits of the Application of PODM to the Design of Interaction}

Because the PODM application project was following a case study project, students were provided with enough background information to understand the dilemma in an interface development process. While the guidance was clearly prescribed and was based on the parameters of an interaction interface, it became much easier for design students to create their first interaction design works. The produced prototypes were published in exe format and sent out to potential users for design evaluation. Although the effectiveness of design was hard to verify due to the small size of sampling, design students got a lot of feedback and insights for future design improvement.

When asked to respond on a five-point Likert scale to broader questions about this design methodology in an end-semester learning evaluation, most students either strongly agreed or agreed that the PODM is great for training decision-making process, useful for guiding project actions and enhancing their understanding of Interaction Design. Although a relevant and effective interaction design may take more effort and time for design testing and refinement, the design students in this class have built strong confidences in design exploration and decision-making, preparing them for the future careers. 


\section{Summary}

As mentioned before, without more detailed knowledge of the effects of the execution of interaction design, design principles can only be applied intuitively, which is difficult for design students. As a result of applying Parameter-Oriented Design Methodology to the projects conducted by design students, it is clear that more precisely defined parameters for the form of interaction design are needed in order to create distinct possibilities aimed toward a target experience. Besides, the integrated approach-combining user, content, and form in the PODM should be comprehensive enough to guide design students or practical designers to make relevant design decisions more effectively. Even so, more work needs to be done- the continuous researches on how to convert intuition into significant knowledge to make interaction design education more effective are of enormous need in this digital era. Design educators are now at the leading edge of a wave of not only technological but also educational change that will affect all aspects of how a product can be designed in a profound way.

\section{References}

1. Mak, B.: Tomorrow's Design Landscape: Anywhere Interactions. Innovation Journal, IDSA, Dulles, 43-49 (2009)

2. Moggridge, B.: Designing Interactions. The MIT Press, Massachusetts (2007)

3. Blair-Early, A., Zender, M.: User Interface Design Principles for Interaction Design, Design Issues, vol. 24(1), pp. 85-107. The MIT Press, Massachusetts (2008)

4. Apple Computer Inc.: Macintosh Human Interface Guidelines. Addison-Wesley, Boston (1992)

5. Tufte, E.: Visual Display of Quantitative Information and Visual Explanations. Graphic Press, CT (1997)

6. Cooper, A., Reimann, R.: About Face 2. Wiley, Boston (2003)

7. van Duyne, D.K., Landay, J.A., Hong, J.I.: The Design of Sites. Addidon-Wesley, Boston (2003)

8. Tognazzini, B.: Maximizing Human Performance, Nielsen Norman Group, http: / / www.asktog.com/basics/03 Performance.html

9. Nielson Norman Group: Site Map Usability, Fremont, California (1998)

10. McCrindle, M.: New Generations at Work: Attracting, Recruiting, Retraining\& Training Generation Y, p.7. McCrindle Research, Sydney (2006)

11. Frand, J.L.: The Information-Age Mindset: Changes in Students and Implications for Higher Education. EDUCAUSE Review 35, 15-24 (2000)

12. Prensky, M.: Digital Natives, Digital Immigrants, pp. 34, On the Horizon (2001)

13. Befooty, C.: What are the Advantages and Disadvantages of Analog vs. Digital Communication?,

http://www.answerbag.com/q_view/31169\#ixzz12pAElagr 